\title{
I-Lacunary Statistical Convergence of Sequences of Sets
}

\author{
Uğur Ulusua ${ }^{\mathrm{a}}$, Erdinç Dündarb \\ ${ }^{a}$ Department of Mathematics, Faculty of Science and Literature, Afyon Kocatepe University, Afyonkarahisar, Turkey \\ ${ }^{b}$ Department of Mathematics, Faculty of Science and Literature, Afyon Kocatepe University, Afyonkarahisar, Turkey
}

\begin{abstract}
In this paper we study the concepts of Wijsman $I$-statistical convergence, Wijsman $\mathcal{I}$-lacunary statistical convergence and Wijsman strongly $\mathcal{I}$-lacunary convergence of sequences of sets and investigate the relationship between them.
\end{abstract}

\section{Introduction, Definitions and NotationS}

The concept of $\mathcal{I}$-convergence in a metric space, which is a generalized from of statistical convergence by Fridy [6], was introduced by Kostyrko, Šalát and Wilczyńki [10]. Later it was further studied many others. Recently, Das et al. [5] introduced new notions, namely $\mathcal{I}$-statistical convergence and $\mathcal{I}$-lacunary statistical convergence by using ideal.

The concept of convergence of sequences of numbers has been extended by several authors to convergence of sequences of sets. The one of these such extensions considered in this paper is the concept of Wijsman convergence (see, [2, 4, 8, 12, 14-16]). Nuray and Rhoades [12] extended the notion of convergence of set sequences to statistical convergence, and gave some basic theorems. Ulusu and Nuray [14] defined the Wijsman lacunary statistical convergence of sequence of sets, and considered its relation with Wiijsman statistical convergence, which was defined by Nuray and Rhoades. Recently, Kişi and Nuray [8] introduced a new convergence notion, for sequences of sets which is called Wijsman $\mathcal{I}$-convergence.

In this paper we extend the concepts of $\mathcal{I}$-statistical convergence and $\mathcal{I}$-lacunary statistical convergence to the concepts of Wijsman $\mathcal{I}$-statistical convergence, Wijsman $\mathcal{I}$-lacunary statistical convergence and Wijsman strongly $I$-lacunary convergence of sequences of sets and investigate the relationship between them which were defined by Kişi and Nuray [8].

2010 Mathematics Subject Classification. Primary 40A05, 40A35

Keywords. Statistical convergence, lacunary sequence, $I$-convergence, sequence of sets, Wijsman convergence.

Received: 17 July 2013; Accepted: 30 July 2013

Communicated by Eberhard Malkowsky

Email addresses: ulusu@aku.edu.tr (Uğur Ulusu), erdincdundar79@gmail.com, edundar@aku.edu.tr (Erdinç Dündar) 


\section{Definitions and Notations}

Let $(X, \rho)$ be a metric space. For any point $x \in X$ and any non-empty subset $A$ of $X$, we define the distance from $x$ to $A$ by

$$
d(x, A)=\inf _{a \in A} \rho(x, a)
$$

Definition 2.1. ([2]) Let $(X, \rho)$ be a metric space. For any non-empty closed subsets $A, A_{k} \subseteq X$, we say that the sequence $\left\{A_{k}\right\}$ is Wijsman convergent to $A$ if

$$
\lim _{k \rightarrow \infty} d\left(x, A_{k}\right)=d(x, A)
$$

for each $x \in X$. In this case we write $W-\lim A_{k}=A$.

Definition 2.2. ([6]) The sequence $x=\left(x_{k}\right)$ of elements of $\mathbb{R}$ is said to be statistically convergent to the number $L$ if for every $\varepsilon>0$,

$$
\lim _{n} \frac{1}{n}\left|\left\{k \leq n:\left|x_{k}-L\right| \geq \varepsilon\right\}\right|=0
$$

In this case we write st $-\lim x_{k}=L$.

Definition 2.3. ([12]) Let $(X, \rho)$ be a metric space. For any non-empty closed subsets $A, A_{k} \subseteq X$, we say that the sequence $\left\{A_{k}\right\}$ is Wijsman statistical convergent to $A$ if $\left\{d\left(x, A_{k}\right)\right\}$ is statistically convergent to $d(x, A)$; i.e., for $\varepsilon>0$ and for each $x \in X$,

$$
\lim _{n \rightarrow \infty} \frac{1}{n}\left|\left\{k \leq n:\left|d\left(x, A_{k}\right)-d(x, A)\right| \geq \varepsilon\right\}\right|=0 .
$$

In this case we write st $-\lim _{W} A_{k}=A$ or $A_{k} \rightarrow A(W S)$.

By a lacunary sequence we mean an increasing integer sequence $\theta=\left\{k_{r}\right\}$ such that $k_{0}=0$ and $h_{r}=k_{r}-k_{r-1} \rightarrow$ $\infty$ as $r \rightarrow \infty$. Throughout this paper the intervals determined by $\theta$ will be denoted by $I_{r}=\left(k_{r-1}, k_{r}\right]$, and ratio $\frac{k_{r}}{k_{r-1}}$ will be abbreviated by $q_{r}$.

Definition 2.4. ([14]) Let $(X, \rho)$ a metric space and $\theta=\left\{k_{r}\right\}$ be a lacunary sequence. For any non-empty closed subsets $A, A_{k} \subseteq X$, we say that the sequence $\left\{A_{k}\right\}$ is Wijsman lacunary statistical convergent to $A$ if $\left\{d\left(x, A_{k}\right)\right\}$ is lacunary statistically convergent to $d(x, A)$; i.e., for $\varepsilon>0$ and for each $x \in X$,

$$
\lim _{r} \frac{1}{h_{r}}\left|\left\{k \in I_{r}:\left|d\left(x, A_{k}\right)-d(x, A)\right| \geq \varepsilon\right\}\right|=0
$$

In this case we write $S_{\theta}-\lim _{W} A_{k}=A$ or $A_{k} \rightarrow A\left(W S_{\theta}\right)$.

Definition 2.5. ([10]) A family of sets $\mathcal{I} \subseteq 2^{\mathbb{N}}$ is called an ideal if and only if 
(i) $\emptyset \in \mathcal{I}$,

(ii) For each $A, B \in \mathcal{I}$ we have $A \cup B \in \mathcal{I}$,

(iii) For each $A \in \mathcal{I}$ and each $B \subseteq A$ we have $B \in \mathcal{I}$.

An ideal is called non-trivial if $\mathbb{N} \notin \mathcal{I}$ and non-trivial ideal is called admissible if $\{n\} \in \mathcal{I}$ for each $n \in \mathbb{N}$.

Definition 2.6. ([10]) A family of sets $F \subseteq 2^{\mathbb{N}}$ is a filter if and only if

(i) $\emptyset \notin F$,

(ii) For each $A, B \in F$ we have $A \cap B \in F$,

(iii) For each $A \in F$ and each $B \supseteq A$ we have $B \in F$.

Proposition 2.7. ([10]) I is a non-trivial ideal in $\mathbb{N}$ if and only if

$$
F(\mathcal{I})=\{M \subset \mathbb{N}:(\exists A \in \mathcal{I})(M=\mathbb{N} \backslash A)\}
$$

is a filter in $\mathbb{N}$.

Definition 2.8. ([10]) An admissible ideal $\mathcal{I} \subset 2^{\mathbb{N}}$ satisfies the property $(A P)$, if for every countable family of mutually disjoint sets $\left\{A_{1}, A_{2}, \ldots\right\}$ belonging to $\mathcal{I}$, there exists a countable family of sets $\left\{B_{1}, B_{2}, \ldots\right\}$ such that $A_{j} \Delta B_{j}$ is a finite set for $j \in \mathbb{N}$ and $B=\bigcup_{j=1}^{\infty} B_{j} \in \mathcal{I}$ (hence $B_{j} \in \mathcal{I}$ for each $j \in \mathbb{N}$ ).

Definition 2.9. ([10]) Let $I \subset 2^{\mathbb{N}}$ be an admissible ideal of subsets of $\mathbb{N}$. A sequence $\left(x_{k}\right)$ of elements of $\mathbb{R}$ is said to be $\mathcal{I}$-convergent to $L \in \mathbb{R}$ if for each $\varepsilon>0$ the set $A(\varepsilon)=\left\{n \in \mathbb{N}:\left|x_{n}-L\right| \geq \varepsilon\right\}$ belongs to $\mathcal{I}$.

Definition 2.10. ([8]) Let $(X, \rho)$ be a metric space and $\mathcal{I} \subseteq 2^{\mathbb{N}}$ be an admissible ideal of subsets of $\mathbb{N}$. For any non-empty closed subsets $A, A_{k} \subset X$, we say that the sequence $\left\{A_{k}\right\}$ is Wijsman $\mathcal{I}$-convergent to $A$, iffor each $\varepsilon>0$ and for each $x \in X$ the set $A(x, \varepsilon)=\left\{k \in \mathbb{N}:\left|d\left(x, A_{k}\right)-d(x, A)\right| \geq \varepsilon\right\}$ belongs to $\mathcal{I}$. In this case we write $\mathcal{I}_{W}-\lim A_{k}=A$ or $A_{k} \rightarrow A\left(\mathcal{I}_{W}\right)$.

Definition 2.11. ([5]) Let $\mathcal{I}$ be an admissible ideal of subsets of $\mathbb{N}$. A sequence $x=\left(x_{k}\right)$ of elements of $\mathbb{R}$ is said to be $\mathcal{I}$-statistically convergent to $L$ or $S(\mathcal{I})$-convergent to $L$ if for each $\varepsilon>0$ and $\delta>0$,

$$
\left\{n \in \mathbb{N}: \frac{1}{n}|k \leq n:| x_{k}-L|\geq \varepsilon| \geq \delta\right\}
$$

belongs to $\mathcal{I}$. In this case, we write $x_{k} \rightarrow L(S(\mathcal{I}))$.

Definition 2.12. ([5]) Let $\theta$ be a lacunary sequence and $\mathcal{I}$ be an admissible ideal of subsets of $\mathbb{N}$. A sequence $x=\left(x_{k}\right)$ of elements of $\mathbb{R}$ is said to be $\mathcal{I}$-lacunary statistically convergent to $L$ or $S_{\theta}(\mathcal{I})$-convergent to $L$ if for each $\varepsilon>0$ and $\delta>0$,

$$
\left\{r \in \mathbb{N}: \frac{1}{h_{r}}\left|k \in I_{r}:\right| x_{k}-L|\geq \varepsilon| \geq \delta\right\}
$$

belongs to $\mathcal{I}$. In this case, we write $x_{k} \rightarrow L\left(S_{\theta}(\mathcal{I})\right)$. 
Definition 2.13. ([5]) Let $\theta$ be a lacunary sequence and $I$ be an admissible ideal of subsets of $\mathbb{N}$. A sequence $x=\left(x_{k}\right)$ of elements of $\mathbb{R}$ is said to be strongly $\mathcal{I}$-lacunary convergent to $L$ or $N_{\theta}(\mathcal{I})$-convergent to $L$ if for each $\varepsilon>0$,

$$
\left\{r \in \mathbb{N}: \frac{1}{h_{r}} \sum_{k \in I_{r}}\left|x_{k}-L\right| \geq \varepsilon\right\}
$$

belong to $\mathcal{I}$. In this case, we write $x_{k} \rightarrow L\left(N_{\theta}(\mathcal{I})\right)$.

Definition 2.14. ([9]) Let $(X, \rho)$ be a metric space and $\mathcal{I} \subseteq 2^{\mathbb{N}}$ be an admissible ideal of subsets of $\mathbb{N}$. For any non-empty closed subsets $A, A_{k} \subset X$, we say that the sequence $\left\{A_{k}\right\}$ is Wijsman $\mathcal{I}$-statistical convergent to $A$ or $S\left(\mathcal{I}_{W}\right)$-convergent to $A$ if for each $\varepsilon>0, \delta>0$ and for each $x \in X$,

$$
\left\{n \in \mathbb{N}: \frac{1}{n}\left|\left\{k \leq n:\left|d\left(x, A_{k}\right)-d(x, A)\right| \geq \varepsilon\right\}\right| \geq \delta\right\}
$$

belongs to $\mathcal{I}$. In this case, we write $A_{k} \rightarrow A\left(S\left(\mathcal{I}_{W}\right)\right)$.

Definition 2.15. ([9]) Let $(X, \rho)$ be a metric space, $\theta$ be lacunary sequence and $I \subseteq 2^{\mathbb{N}}$ be an admissible ideal of subsets of $\mathbb{N}$. For any non-empty closed subsets $A, A_{k} \subset X$, we say that the sequence $\left\{A_{k}\right\}$ is Wijsman $I$-lacunary statistical convergent to $A$ or $S_{\theta}\left(\mathcal{I}_{W}\right)$-convergent to $A$ if for each $\varepsilon>0, \delta>0$ and for each $x \in X$,

$$
\left\{r \in \mathbb{N}: \frac{1}{h_{r}}\left|\left\{k \in I_{r}:\left|d\left(x, A_{k}\right)-d(x, A)\right| \geq \varepsilon\right\}\right| \geq \delta\right\}
$$

belongs to $\mathcal{I}$. In this case, we write $A_{k} \rightarrow A\left(S_{\theta}\left(\mathcal{I}_{W}\right)\right)$.

Definition 2.16. ([9]) Let $(X, \rho)$ be a metric space, $\theta$ be lacunary sequence and $\mathcal{I} \subseteq 2^{\mathbb{N}}$ be an admissible ideal of subsets of $\mathbb{N}$. For any non-empty closed subsets $A, A_{k} \subset X$, we say that the sequence $\left\{A_{k}\right\}$ is said to be Wijsman strongly $\mathcal{I}$-lacunary convergent to $A$ or $N_{\theta}\left[\mathcal{I}_{W}\right]$-convergent to $A$ if for each $\varepsilon>0$ and for each $x \in X$,

$$
\left\{r \in \mathbb{N}: \frac{1}{h_{r}} \sum_{k \in I_{r}}\left|d\left(x, A_{k}\right)-d(x, A)\right| \geq \varepsilon\right\}
$$

belongs to $\mathcal{I}$. In this case, we write $A_{k} \rightarrow A\left(N_{\theta}\left[\mathcal{I}_{W}\right]\right)$.

\section{Main Results}

In this section, we investigate the relationship between the concepts of Wijsman $\mathcal{I}$-statistical convergence, Wijsman $\mathcal{I}$-lacunary statistical convergence and Wijsman strongly $\mathcal{I}$-lacunary convergence of sequences of sets.

Theorem 3.1. Let $(X, \rho)$ be a metric space, $\theta=\left\{k_{r}\right\}$ be a lacunary sequence, $I \subseteq 2^{\mathbb{N}}$ be an admissible ideal and $A, A_{k}$ be non-empty closed subsets of $X$. Then, $A_{k} \rightarrow A\left(N_{\theta}\left[\mathcal{I}_{W}\right]\right) \Rightarrow A_{k} \rightarrow A\left(S_{\theta}\left(\mathcal{I}_{W}\right)\right)$. 
Proof. Let $A_{k} \rightarrow A\left(N_{\theta}\left[\mathcal{I}_{W}\right]\right)$ and $\varepsilon>0$. Then, for each $x \in X$ we can write

$$
\begin{aligned}
\sum_{k \in I_{r}}\left|d\left(x, A_{k}\right)-d(x, A)\right| & \geq \sum_{\substack{k \in I_{r} \\
\left|d\left(x, A_{k}\right)-d(x, A)\right| \geq \varepsilon}}\left|d\left(x, A_{k}\right)-d(x, A)\right| \\
& \geq \varepsilon \cdot\left|\left\{k \in I_{r}:\left|d\left(x, A_{k}\right)-d(x, A)\right| \geq \varepsilon\right\}\right|
\end{aligned}
$$

and so

$$
\frac{1}{\varepsilon \cdot h_{r}} \sum_{k \in I_{r}}\left|d\left(x, A_{k}\right)-d(x, A)\right| \geq \frac{1}{h_{r}}\left|\left\{k \in I_{r}:\left|d\left(x, A_{k}\right)-d(x, A)\right| \geq \varepsilon\right\}\right| .
$$

Hence, for each $x \in X$ and for any $\delta>0$,

$$
\left\{r \in \mathbb{N}: \frac{1}{h_{r}}\left|\left\{k \in I_{r}:\left|d\left(x, A_{k}\right)-d(x, A)\right| \geq \varepsilon\right\}\right| \geq \delta\right\} \subseteq\left\{r \in \mathbb{N}: \frac{1}{h_{r}} \sum_{k \in I_{r}}\left|d\left(x, A_{k}\right)-d(x, A)\right| \geq \varepsilon \cdot \delta\right\} \in \mathcal{I} .
$$

This proof is comleted.

Theorem 3.2. Let $(X, \rho)$ be a metric space, $\theta=\left\{k_{r}\right\}$ be a lacunary sequence, $I \subseteq 2^{\mathbb{N}}$ be an admissible ideal and $A, A_{k}$ be non-empty closed subsets of $X$. Then, $\left\{A_{k}\right\} \in L_{\infty}$ and $A_{k} \rightarrow A\left(S_{\theta}\left(\mathcal{I}_{W}\right)\right) \Rightarrow A_{k} \rightarrow A\left(N_{\theta}\left[\mathcal{I}_{W}\right]\right)$.

Proof. Suppose that $A_{k} \rightarrow A\left(S_{\theta}\left(\mathcal{I}_{W}\right)\right)$ and $A_{k} \in L_{\infty}$. Then there exists an $M>0$ such that $\left|d\left(x, A_{k}\right)-d(x, A)\right| \leq M$ for each $x \in X$ and all $k \in \mathbb{N}$. Given $\varepsilon>0$, for each $x \in X$ we have

$$
\begin{aligned}
\frac{1}{h_{r}} \sum_{k \in I_{r}}\left|d\left(x, A_{k}\right)-d(x, A)\right| & =\frac{1}{h_{r}} \sum_{\substack{k \in I_{r} \\
\left|d\left(x, A_{k}\right)-d(x, A)\right| \geq \frac{\varepsilon}{2}}}\left|d\left(x, A_{k}\right)-d(x, A)\right|+\frac{1}{h_{r}} \sum_{\substack{k \in I_{r} \\
\left|d\left(x, A_{k}\right)-d(x, A)\right|<\frac{\varepsilon}{2}}}\left|d\left(x, A_{k}\right)-d(x, A)\right| \\
& \leq \frac{M}{h_{r}}\left|\left\{k \in I_{r}:\left|d\left(x, A_{k}\right)-d(x, A)\right| \geq \frac{\varepsilon}{2}\right\}\right|+\frac{\varepsilon}{2} .
\end{aligned}
$$

Hence, for each $x \in X$ we have

$$
\left\{r \in \mathbb{N}: \frac{1}{h_{r}} \sum_{k \in I_{r}}\left|d\left(x, A_{k}\right)-d(x, A)\right| \geq \varepsilon\right\} \subseteq\left\{r \in \mathbb{N}: \frac{1}{h_{r}}\left|\left\{k \in I_{r}:\left|d\left(x, A_{k}\right)-d(x, A)\right| \geq \frac{\varepsilon}{2}\right\}\right| \geq \frac{\varepsilon}{2 M}\right\} \in \mathcal{I} .
$$

This proof is completed.

We have the following Theorem by Theorem 3.1 and Theorem 3.2.

Theorem 3.3. Let $(X, \rho)$ be a metric space, $\theta=\left\{k_{r}\right\}$ be a lacunary sequence, $I \subseteq 2^{\mathbb{N}}$ be an admissible ideal and $A, A_{k}$ be non-empty closed subsets of $X$. Then, $S_{\theta}\left(\mathcal{I}_{W}\right) \cap L_{\infty}=N_{\theta}\left[\mathcal{I}_{W}\right] \cap L_{\infty}$, where $S_{\theta}\left(\mathcal{I}_{W}\right), N_{\theta}\left[\mathcal{I}_{W}\right]$ and $L_{\infty}$ denote the sets of Wijsman I-lacunary statistical convergent sequences, Wijsman strongly $\mathcal{I}$ - lacunary convergent sequences and bounded sequences of sets, respectively.

Theorem 3.4. Let $(X, \rho)$ be a metric space, $\theta=\left\{k_{r}\right\}$ be a lacunary sequence, $I \subseteq 2^{\mathbb{N}}$ be an admissible ideal and $A, A_{k}$ be non-empty closed subsets of $X$. Then, $A_{k} \rightarrow A\left(S\left(\mathcal{I}_{W}\right)\right)$ implies $A_{k} \rightarrow A\left(S_{\theta}\left(\mathcal{I}_{W}\right)\right)$ if and only if $\lim _{i n f} q_{r}>1$. If $\liminf _{r} q_{r}=1$, then there exists a bounded sequence $\left\{A_{k}\right\}$ which is Wijsman $\mathcal{I}$-statistical convergent but not Wijsman I-lacunary statistical convergent. 
Proof. Suppose first that $\lim \inf _{r} q_{r}>1$; then there exists a $\lambda>0$ such that $q_{r} \geq 1+\lambda$ for sufficiently large $r$, which implies that

$$
\frac{h_{r}}{k_{r}} \geq \frac{\lambda}{1+\lambda} \text {. }
$$

If $A_{k} \rightarrow A\left(S\left(\mathcal{I}_{W}\right)\right.$, then for every $\varepsilon>0$, for each $x \in X$ and for sufficiently large $r$, we have

$$
\begin{aligned}
\frac{1}{k_{r}}\left|\left\{k \leq k_{r}:\left|d\left(x, A_{k}\right)-d(x, A)\right| \geq \varepsilon\right\}\right| & \geq \frac{1}{k_{r}}\left|\left\{k \in I_{r}:\left|d\left(x, A_{k}\right)-d(x, A)\right| \geq \varepsilon\right\}\right| \\
& \geq \frac{\lambda}{1+\lambda} \cdot\left(\frac{1}{h_{r}}\left|\left\{k \in I_{r}:\left|d\left(x, A_{k}\right)-d(x, A)\right| \geq \varepsilon\right\}\right|\right) .
\end{aligned}
$$

Hence, for each $x \in X$ and for any $\delta>0$ we have

$$
\left\{r \in \mathbb{N}: \frac{1}{h_{r}}\left|\left\{k \in I_{r}:\left|d\left(x, A_{k}\right)-d(x, A)\right| \geq \varepsilon\right\}\right| \geq \delta\right\} \subseteq\left\{r \in \mathbb{N}: \frac{1}{k_{r}}\left|\left\{k \leq k_{r}:\left|d\left(x, A_{k}\right)-d(x, A)\right| \geq \varepsilon\right\}\right| \geq \frac{\delta \lambda}{(1+\lambda)}\right\} \in \mathcal{I} .
$$

This proves the sufficiency.

Conversely, suppose $\liminf _{r} q_{r} \leq 1$. Since $k_{r}$ is nondecreasing we have $q_{r}=k_{r} / k_{r-1} \geq 1$ and $\liminf _{r} q_{r} \geq 1$. Then, we have $\lim _{\inf } q_{r}=1$. In the following we will proceed as in [5]. Since $\theta$ is lacunary, we can select a subsequence $\left\{k_{r_{j}}\right\}$ of lacunary sequence $\theta$ such that

$$
\frac{k_{r_{j}}}{k_{r_{j}-1}}<1+\frac{1}{j} \quad \text { and } \quad \frac{k_{r_{j}-1}}{k_{r_{j-1}}}>j, \quad \text { where } r_{j} \geq r_{j-1}+2 .
$$

Now we define a sequence $\left\{A_{k}\right\}$ as follows:

$$
A_{k}:= \begin{cases}(x, y) \in \mathbb{R}^{2}, x^{2}+(y-1)^{2}=\frac{1}{k^{4}}, & k \in I_{r_{j},} \\ \{(0,0)\} & \text { otherwise. }\end{cases}
$$

Then, for at least one $x \in X$, we have

$$
\frac{1}{h_{r_{j}}} \sum_{k \in I_{r_{j}}}\left|d\left(x, A_{k}\right)-d(x,\{(0,0)\})\right|=T, \quad \text { for } j=1,2, \cdots,\left(T \in \mathbb{R}^{+}\right)
$$

and

$$
\frac{1}{h_{r}} \sum_{k \in I_{r}}\left|d\left(x, A_{k}\right)-d(x,\{(0,0)\})\right|=0, \quad \text { for } r \neq r_{j} .
$$

Then, it is quite clear that $\left\{A_{k}\right\} \notin N_{\theta}\left[\mathcal{I}_{W}\right]$. Since $\left\{A_{k}\right\}$ is bounded, by Theorem $3.3\left\{A_{k}\right\} \notin\left(S_{\theta}\left(I_{W}\right)\right)$.

Now, let $k_{r_{j}-1}<n<k_{r_{j+1}-1}$. Then, for each $\varepsilon>0$ and each $x \in X$ we have

$$
\frac{\varepsilon}{n}\left|\left\{k \leq n:\left|d\left(x, A_{k}\right)-d(x,\{(0,0)\})\right| \geq \varepsilon\right\}\right| \leq \frac{1}{n} \sum_{k=1}^{n}\left|d\left(x, A_{k}\right)-d(x,\{(0,0)\})\right| \leq \frac{k_{r_{j-1}}+h_{r_{j}}}{k_{r_{j}-1}} \leq \frac{1}{j}+\frac{1}{j}=\frac{2}{j} .
$$

As $n \rightarrow \infty$ it follows that also $j \rightarrow \infty$. Hence, $\left\{A_{k}\right\} \in\left(S\left(\mathcal{I}_{W}\right)\right)$. This proof is completed.

Theorem 3.5. Let $(X, \rho)$ be a metric space, $\mathcal{I} \subseteq 2^{\mathbb{N}}$ be an admissible ideal satisfying property $(A P), \theta \in F(\mathcal{I})$ and $A, A_{k}$ be non-empty closed subsets of X. If $\left\{A_{k}\right\} \in\left(S\left(\mathcal{I}_{W}\right)\right) \cap\left(S_{\theta}\left(\mathcal{I}_{W}\right)\right)$, then $S\left(\mathcal{I}_{W}\right)-\lim A_{k}=S_{\theta}\left(\mathcal{I}_{W}\right)-\lim A_{k}$. 
Proof. Suppose that $S\left(\mathcal{I}_{W}\right)-\lim A_{k}=A$ and $S_{\theta}\left(\mathcal{I}_{W}\right)-\lim A_{k}=B$ and $A \neq B$. Let $0<\varepsilon<\frac{1}{2}|d(x, A)-d(x, B)|$ for each $x \in X$. Since $\mathcal{I}$ satisfies the property $(A P)$, there exists $M \in F(\mathcal{I})(i . e ., \mathbb{N} \backslash M \in \mathcal{I}$ ) such that for each $x \in X$,

$$
\lim _{r \rightarrow \infty} \frac{1}{m_{r}}\left|\left\{k \leq m_{r}:\left|d\left(x, A_{k}\right)-d(x, A)\right| \geq \varepsilon\right\}\right|=0, \quad \text { where } M=\left\{m_{1}, m_{2}, m_{3}, \ldots\right\} .
$$

Let

$$
P=\left\{k \leq m_{r}:\left|d\left(x, A_{k}\right)-d(x, A) \geq \varepsilon\right|\right\} \text { and } R=\left\{k \leq m_{r}:\left|d\left(x, A_{k}\right)-d(x, B)\right| \geq \varepsilon\right\} .
$$

Then $m_{r}=|P \cup R| \leq|P|+|R|$. This implies that $1 \leq \frac{|P|}{m_{r}}+\frac{|R|}{m_{r}}$. Since $\frac{|R|}{m_{r}} \leq 1$ and $\lim _{r \rightarrow \infty} \frac{|P|}{m_{r}}=0$, so we must have

$$
\lim _{r \rightarrow \infty} \frac{|R|}{m_{r}}=1
$$

Let $M^{*}=\left\{k_{l_{1}}, k_{l_{2}}, k_{l_{3}}, \ldots\right\}=M \cap \theta \in F(\mathcal{I})$.

Then the $k_{l_{p}}$ th term of the statistical limit expression $\frac{1}{m_{r}}\left|\left\{k \leq m_{r}:\left|d\left(x, A_{k}\right)-d(x, B)\right| \geq \varepsilon\right\}\right|$ is

$$
\frac{1}{k_{l_{p}}}\left|\left\{k \in \bigcup_{i=1}^{l_{p}} I_{i}:\left|d\left(x, A_{k}\right)-d(x, B)\right| \geq \varepsilon\right\}\right|=\frac{1}{\sum_{i=1}^{l_{p}} h_{i}} \sum_{i=1}^{l_{p}} t_{i} h_{i},
$$

where $t_{i}=\frac{1}{h_{i}}\left|\left\{k \in I_{i}:\left|d\left(x, A_{k}\right)-d(x, B)\right| \geq \varepsilon\right\}\right| \stackrel{\mathcal{I}}{\rightarrow} 0$ because $\left\{A_{k}\right\} \rightarrow B\left(S_{\theta}\left(\mathcal{I}_{W}\right)\right)$. Since $\theta$ is a lacunary sequence, (1) is a regular weighted mean transform of $t_{i}$ 's and therefore it is also $\mathcal{I}$-convergent to 0 as $p \rightarrow \infty$, and so it has a subsequence which is convergent to 0 since $I$ satisfies property $(A P)$. But since this is a subsequence of $\left\{\frac{1}{n}\left|\left\{k \leq n:\left|d\left(x, A_{k}\right)-d(x, B)\right| \geq \varepsilon\right\}\right|\right\}_{n \in M}$, we infer that $\left\{\frac{1}{n}\left|\left\{k \leq n:\left|d\left(x, A_{k}\right)-d(x, B)\right| \geq \varepsilon\right\}\right|\right\}_{n \in M}$ is not convergent to 1 . This is a contradiction. Hence the proof is completed.

\section{Acknowledgment}

The authors are grateful to the referees for their careful reading of the article and their valuable suggestions.

\section{References}

[1] J.-P. Aubin, H. Frankowska, Set-valued analysis, Birkhauser, Boston (1990).

[2] M. Baronti, P. Papini, Convergence of sequences of sets, In: Methods of functional analysis in approximation theory, ISNM 76, Birkhauser-Verlag, Basel (1986).

[3] G. Beer, On convergence of closed sets in a metric space and distance functions, Bull. Aust. Math. Soc. 31 (1985) $421-432$.

[4] G. Beer, Wijsman convergence: A survey, Set-Valued Var. Anal. 2 (1994) 77-94.

[5] P. Das, E. Savaş, S. Kr. Ghosal, On generalized of certain summability methods using ideals, Appl. Math. Letter 36 (2011) 1509-1514.

[6] J. A. Fridy, On statistical convergence, Analysis 5 (1985) 301-313.

[7] J. A. Fridy, C. Orhan, Lacunary Statistical Convergence, Pacific J. Math. 160(1) (1993) 43-51.

[8] Ö. Kişi, F. Nuray, A new convergence for sequences of sets, Abstr. Appl. Anal., Vol.2013 (2013) Article ID 852796, 6 pages.

[9] Ö. Kişi, E. Savaş, F. Nuray, On $I$-asymptotically lacunary statistical equivalence of sequences of sets, (submitted for publication).

[10] P. Kostyrko, T. Šalát, W. Wilczyński, I-Convergence, Real Anal. Exchange 26(2) (2000) 669-686. 
[11] P. Kostyrko, M. Mačaj, T. Šalát, M. Sleziak, I-convergence and extremal I-limit points, Math. Slovaca 55 (2005) 443-464.

[12] F. Nuray, B. E. Rhoades, Statistical convergence of sequences of sets, Fasc. Math. 49 (2012) 87-99.

[13] E. Savaş, P. Das, A generalized statistical convergence via ideals, Appl. Math. Letters 24 (2011) 826-830.

[14] U. Ulusu, F. Nuray, Lacunary statistical convergence of sequence of sets, Progress in Applied Mathematics 4(2) (2012) 99-109.

[15] R. A. Wijsman, Convergence of sequences of convex sets, cones and functions, Bull. Amer. Math. Soc. 70 (1964) 186-188.

[16] R. A. Wijsman, Convergence of Sequences of Convex sets, Cones and Functions II, Trans. Amer. Math. Soc. 123(1) (1966) 32-45. 